\title{
A new sensitive method for the quantification of glyoxal and methylglyoxal in snow and ice by stir bar sorptive extraction and liquid desorption-HPLC-ESI-MS
}

\author{
Christina Müller-Tautges • Anja Eichler • \\ Margit Schwikowski • Thorsten Hoffmann
}

Received: 8 November 2013 /Revised: 17 January 2014 / Accepted: 20 January 2014 / Published online: 9 February 2014

(C) Springer-Verlag Berlin Heidelberg 2014

\begin{abstract}
In this study, the development of a new sensitive method for the analysis of alpha-dicarbonyls glyoxal $(\mathrm{G})$ and methylglyoxal (MG) in environmental ice and snow is presented. Stir bar sorptive extraction with in situ derivatization and liquid desorption (SBSE-LD) was used for sample extraction, enrichment, and derivatization. Measurements were carried out using high-performance liquid chromatography coupled to electrospray ionization tandem mass spectrometry (HPLC-ESI-MS/MS). As part of the method development, SBSE-LD parameters such as extraction time, derivatization reagent, desorption time and solvent, and the effect of $\mathrm{NaCl}$ addition on the SBSE efficiency as well as measurement parameters of HPLC-ESI-MS/MS were evaluated. Calibration was performed in the range of $1-60 \mathrm{ng} / \mathrm{mL}$ using spiked ultrapure water samples, thus incorporating the complete SBSE and derivatization process. 4-Fluorobenzaldehyde was applied as internal standard. Inter-batch precision was $<12 \%$ RSD. Recoveries were determined by means of spiked snow samples and were $78.9 \pm 5.6 \%$ for $\mathrm{G}$ and $82.7 \pm 7.5 \%$ for $\mathrm{MG}$, respectively. Instrumental detection limits of 0.242 and $0.213 \mathrm{ng} / \mathrm{mL}$ for $\mathrm{G}$ and $\mathrm{MG}$ were achieved using the multiple reaction monitoring mode. Relative detection limits referred to
\end{abstract}

C. Müller-Tautges $\cdot$ T. Hoffmann $(\square)$

Institute of Inorganic and Analytical Chemistry, Johannes

Gutenberg-University, 55122 Mainz, Germany

e-mail: t.hoffmann@uni-mainz.de

A. Eichler $\cdot$ M. Schwikowski

Laboratory of Radiochemistry and Environmental Chemistry, Paul

Scherrer Institute, 5232 Villigen, Switzerland

A. Eichler $\cdot$ M. Schwikowski

Oeschger Centre for Climate Change Research, University of Bern, 3012 Bern, Switzerland

M. Schwikowski

Department for Chemistry and Biochemistry, University of Bern,

3012 Bern, Switzerland a sample volume of $15 \mathrm{~mL}$ were $0.016 \mathrm{ng} / \mathrm{mL}$ for $\mathrm{G}$ and $0.014 \mathrm{ng} / \mathrm{mL}$ for MG. The optimized method was applied for the analysis of snow samples from Mount Hohenpeissenberg (close to the Meteorological Observatory Hohenpeissenberg, Germany) and samples from an ice core from Upper Grenzgletscher (Monte Rosa massif, Switzerland). Resulting concentrations were $0.085-16.3 \mathrm{ng} / \mathrm{mL}$ for $\mathrm{G}$ and $0.126-3.6 \mathrm{ng} / \mathrm{mL}$ for MG. Concentrations of $\mathrm{G}$ and $\mathrm{MG}$ in snow were 1-2 orders of magnitude higher than in ice core samples. The described method represents a simple, green, and sensitive analytical approach to measure $G$ and $M G$ in aqueous environmental samples.

Keywords Glyoxal $\cdot$ Methylglyoxal $\cdot$ SBSE $\cdot$ Liquid desorption · HPLC-MS · Ice

\section{Introduction}

The two smallest alpha-dicarbonyls glyoxal (G) and methylglyoxal (MG) have received increasing scientific interest in recent years due to their important role in the formation and growth of secondary organic aerosol (SOA) [1]. SOA itself is of great interest because of its impact on the radiative budget of the earth's atmosphere [2]. G and MG are formed through gas phase oxidation of volatile organic compounds (VOCs) of both anthropogenic (i.e., aromatics, alkenes) and biogenic (i.e., isoprene) origin [1, 3, 4]. Besides secondary formation, $\mathrm{G}$ and $\mathrm{MG}$ can also be emitted directly into the atmosphere by biomass burning [1]. The lifetime of the alphadicarbonyls in the gas phase is rather short (about $2 \mathrm{~h}$ ) [5]. However, due to their high Henry's law coefficients, G and MG efficiently partition into the (aqueous) particle phase. Besides, the compounds are easily scavenged by precipitation (i.e., fog, cloud droplets, rain, or snow), either directly from the gas phase or as component of aerosol particles $[6,7]$. In the 
aqueous phase, G and MG reversibly form hydrated monomers and oligomers. Through further aqueous phase chemistry (particularly in cloud water), alpha-dicarbonyls can be oxidized by $\mathrm{OH}$ radicals to form organic acids, especially oxalic and pyruvic acid, which are found in SOA [8]. Oligomerization of either $\mathrm{G} / \mathrm{MG}$ or their oxidation products can also contribute to SOA formation $[9,10]$.

In high altitude and latitude regions, snow is the only type of precipitation. After scavenging and deposition, organic compounds in the seasonal snow layer may undergo transformation processes such as physical exchange ("wind pumping"), photochemical production from other precursors or degradation $[11,12]$. Until now, very little is known about the role of the small, water-soluble compounds G and MG in these complex processes. Their determination is of high interest and can possibly give insight into chemical processes occurring in the ice. By analyzing ice core samples, information about the atmospheric composition in the past-interrelated with the climate - is accessible. In addition to studies focusing on the quantification of organic compounds, like for example VOCs, persistent organic pollutants, or carboxylic acids in snow and ice (e.g., [13-15]) there are only few studies targeting $\mathrm{G}$ and $\mathrm{MG}$ in snow and frost flowers ([16-18]), as well as in ice cores ([19]).

The analysis of carbonyl compounds usually comprises a derivatization step. This is crucial regarding GC analyses as it improves volatility and stability of the analytes, for example. A commonly used derivatization approach is pentafluorobenzyl hydroxylamine (PFBHA) derivatization with GC-ECD detection [20]. A study using PFBHA derivatization in combination with GC-MS was recently presented for the analysis of G and MG in seawater and marine aerosol [5]. Derivatization with $\mathrm{BF}_{3} / \mathrm{n}$-butanol, which yields the corresponding dibutyl acetals, was applied for the measurement of $G$ and $M G$ in rain and snow, also using GC and GC-MS ([21]). However, with the continuous development of atmospheric pressure ionization techniques such as electrospray ionization (ESI), the number of applications using LC and LC-MS has increased enormously over the last decades. The possibility of using various column materials, eluent compositions, and detection modes makes LC(-MS) a promising tool to meet the increasing needs of analytical measurements. Although not obligatory, derivatization is a powerful strategy to enhance the capabilities of LC (-MS) in various ways, i.e., concerning chromatographic, ionization, and detection properties [22]. The most frequently used derivatizing reagent for the analysis of carbonyl compounds with LC is 2,4-dinitrophenylhydrazine (DNPH) [23, 24]. A selective derivatization of alpha-dicarbonyls was also achieved using aromatic diamino compounds such as 2,3diaminonaphthalene. The resulting highly fluorescent derivatization products were quantified using fluorescence detection [25]. Dansylacetamidooxyamine derivatization with LCfluorescence detection was used for the highly sensitive analysis of alpha-dicarbonyls and hydroxyacetaldehyde as well as monoaldehydes [17].

The aim of this study was the development of a new simple LC-MS method for the sensitive quantification of $G$ and MG in snow and ice. Stir bar sorptive extraction (SBSE) with in situ derivatization is applied for extraction and enrichment of the analytes. SBSE is an equilibrium technique which uses stir bars with polydimethylsiloxane (PDMS) coating. The extraction is based on the partitioning of the solutes between the siloxane phase (stir bar) and the aqueous sample. In a second step, the analytes are recovered from the stir bar. This is achieved either by thermal desorption using a thermal desorption device attached to a GC system or by liquid desorption (LD). In the latter case, the analytes are desorbed by a solvent with higher elution strength than the original sample. LD is compatible with LC-MS analysis and does not require additional hardware [26]. Compared to solid phase micro extraction, which is based on the same sorptive extraction principle, SBSE requires less technical equipment and can achieve higher extraction efficiency due to a larger volume of the siloxane phase. No time-consuming evaporation of the aqueous sample is needed, as the stir bar can be added directly to the sample. SBSE-LD is a "green" extraction technique, because it is less solvent intensive than liquid-liquid extraction. In contrast to the frequently used solid phase extraction (SPE), SBSE-LD is less labor- and cost-intensive because the stir bars are easy to handle and can be reused multiple times.

To the authors' best knowledge, this method is the first to combine SBSE with in situ benzylhydroxylamine (BHA) derivatization and HPLC-MS/MS for the analysis of G and MG.

\section{Experimental section}

\section{Chemicals and materials}

$\mathrm{G}$ and $\mathrm{MG}(40 \%(w)$ aqueous solutions) were purchased from Acros Organics (Thermo Fisher Scientific, Geel, Belgium). $O$ benzylhydroxylamine hydrochloride (BHA $\cdot \mathrm{HCl}, 99 \%$ ), $O$-(2,3,4,5,6-pentafluorobenzyl) hydroxylamine hydrochloride (PFBHA $\cdot \mathrm{HCl}, \mathrm{GC}$ derivatization grade), 4fluorobenzaldehyde (98\%), sulfuric acid (95-98\%), dichloromethane (HPLC grade), acetonitrile (HPLC gradient grade), and formic acid (LC-MS grade) were purchased from SigmaAldrich (Steinheim, Germany). Hydrochloric acid (30 \%) was obtained from Merck (Darmstadt, Germany). Sodium hydroxide was purchased from Carl Roth (Karlsruhe, Germany). Sodium chloride (HPLC grade) was purchased from Fisher Chemical (Geel, Belgium). Ultrapure water $(18.3 \mathrm{M} \Omega \mathrm{cm})$ was obtained using a Milli-Q water system from Millipore (Bedford, USA). PDMS-coated stir bars (Twister ${ }^{\circledR}$, film thickness $1 \mathrm{~mm}$, length $10 \mathrm{~mm}$ ) were purchased from Gerstel 
(Mülheim, Germany). SPE cartridges (Discovery DSC-18, $1 \mathrm{~mL}, 100 \mathrm{mg}$ bed volume) were purchased from Supelco (Sigma-Aldrich, Steinheim, Germany).

\section{Purification of the derivatization reagent}

$\mathrm{BHA} \cdot \mathrm{HCl}$ was purified adapting a method described by Matsunaga and Kawamura [16]. Briefly, $\mathrm{BHA} \cdot \mathrm{HCl}$ was dissolved in ultrapure water. Sodium hydroxide pellets were flushed with methanol and added to the solution. Free benzylhydroxylamine was extracted with dichloromethane and precipitated as $\mathrm{BHA} \cdot \mathrm{HCl}$ by adding $8 \mathrm{M}$ $\mathrm{HCl}$ solution. The precipitate was filtered and dried under reduced pressure.

A stock solution was prepared by diluting $125 \mathrm{mg}$ purified $\mathrm{BHA} \mathrm{HCl}$ in $20 \mathrm{~mL}$ ultrapure water. The stock solution was stored at $4{ }^{\circ} \mathrm{C}$ and was stable over several months. Prior to sample preparation, an aliquot of the stock solution was purified using SPE with a C18 stationary phase: The SPE cartridge was conditioned with $2 \times 800 \mu \mathrm{L}$ acetonitrile and washed with $2 \times 800 \mu \mathrm{L}$ ultrapure water. Four milliliters of the BHA solution were applied to the cartridge by means of a low pressure to retain possible contaminants. The eluted solution was collected and applied for derivatization.

\section{SBSE procedure}

All glass vials (sample flasks, extraction vials, liquid desorption vials, and storage vials) were cleaned by heating at $450{ }^{\circ} \mathrm{C}$ for $8 \mathrm{~h}$. Prior to use, the stir bars were put in glass vials filled with 2-3 $\mathrm{mL}$ acetonitrile and cleaned in an ultrasonic bath for $30 \mathrm{~min}$ before stirring in $3 \mathrm{~mL}$ fresh acetonitrile at $1,000 \mathrm{rpm}$ overnight. These cleaning steps were repeated after every analysis to avoid carry over.

For extraction, spiked water samples (for calibration) or environmental samples were filled into $20 \mathrm{~mL}$ screw-cap vials with PTFE-septa. One hundred fifty microliters of internal standard solution (4-fluorobenzaldehyde) and $100 \mu \mathrm{L}$ of derivatization solution (BHA) were added. The $\mathrm{pH}$ was adjusted to 3.5 with $0.1 \mathrm{M}$ sulfuric acid. The stir bars were removed from the storage vials using magnetic rods, dried carefully with a lint-free tissue and added to the sample solution. The vials were closed tightly and put on a 15-point magnetic stirring plate for $20 \mathrm{~h}$ at 1,000 rpm. After extraction, stir bars were removed from the extraction solution and transferred into $5 \mathrm{~mL}$ vials containing $1.5 \mathrm{~mL}$ acetonitrile as stripping solvent for liquid desorption. After stirring for $3 \mathrm{~h}$ at $500 \mathrm{rpm}$, the stir bars were removed from the vials. The desorption solution was transferred to an autosampler vial and provided for analysis. Stir bars were cleaned as described above and stored in acetonitrile (ACN) until further use. An overview of the SBSE-LD procedure is shown in Fig. 1. During method development, several important SBSE parameters such as

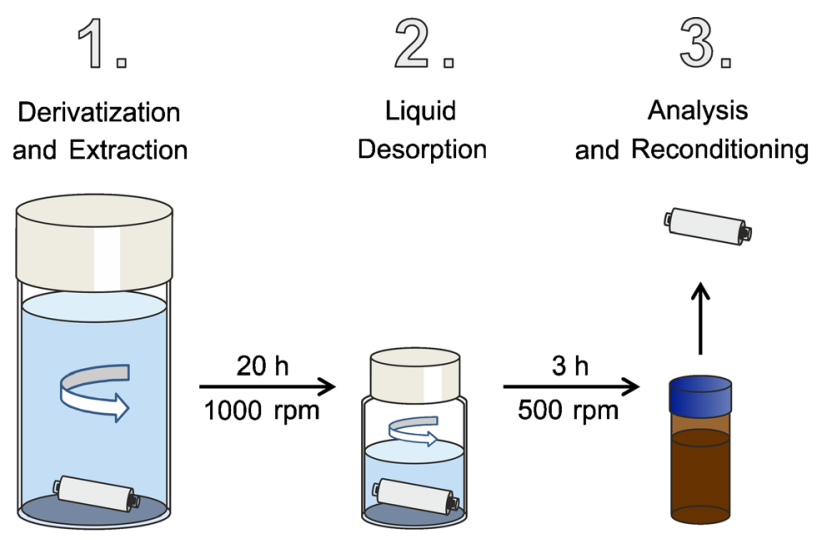

aqueous sample

organic solvent

HPLC/MS sample

extraction time $(2-20 \mathrm{~h})$, liquid desorption time $(0.5-20 \mathrm{~h})$ and solvent (methanol, acetonitrile) were optimized.

\section{Liquid chromatography-mass spectrometry}

The high-performance liquid chromatography coupled to electrospray ionization tandem mass spectrometry (HPLCESI-MS/MS) measurements were carried out using a HPLC system (Agilent 1100 series, composed of degasser, binary gradient pump, auto sampler, column oven, and diode array detector, Agilent Technologies, Germany) coupled to a HCTPlus ion trap mass spectrometer (Bruker-Daltonics, Bremen, Germany). The analytical column (Pursuit XRs 3 C8 150× $2.0 \mathrm{~mm}, 3 \mu \mathrm{m}$ particle size, Varian, Germany) was heated to $30{ }^{\circ} \mathrm{C}$ during analysis.

Eluent A (ultrapure water with $2 \%$ acetonitrile and $0.04 \%$ formic acid) and B (acetonitrile with $2 \%$ ultrapure water) were used in gradient mode with a constant flow rate of $200 \mu \mathrm{L} / \mathrm{min}$. The injection volume was $20 \mu \mathrm{L}$.

The ESI source was operated in positive ion mode with 35 psi nebulizer pressure, $9 \mathrm{~L} / \mathrm{min}$ dry gas flow (both $\mathrm{N}_{2}$ ), $350{ }^{\circ} \mathrm{C}$ dry gas temperature, and $4.5 \mathrm{kV}$ capillary voltage. All other electronic parameters were set using the smart tune option for the respective $\mathrm{m} / \mathrm{z}$ values of the analyte molecular ions. Analysis was performed using the multi reaction monitoring (MRM) mode which significantly reduces background noise: The protonated molecular ions $[\mathrm{M}+\mathrm{H}]^{+}$of the derivatization products ( $\mathrm{m} / \mathrm{z} 269$ and $\mathrm{m} / \mathrm{z} 283$ for glyoxal-bisoxime and methylglyoxal-bis-oxime) were isolated and fragmented in the ion trap using collisional induced dissociation (CID). Helium was used as collision gas with optimized fragmentation amplitude of 0.7 (specific parameter of the instrument). The most abundant fragment ions $(\mathrm{m} / \mathrm{z} 91$ for both glyoxal-bis-oxime and methylglyoxal-bis-oxime) were monitored and used for quantification. 
Standards

Stock solutions of G and MG with concentrations of 506 and $471 \mathrm{ng} / \mathrm{mL}$, respectively, were prepared daily by diluting the commercially available solutions with ultrapure water. To obtain method development and calibration solutions, volumes of $15 \mathrm{~mL}$ ultrapure water were spiked with distinct volumes of $\mathrm{G}$ (or MG) stock solution and $5.7 \mu \mathrm{g} / \mathrm{L}$ of the internal standard. The spiked solutions were treated as described in the "SBSE procedure" section above. That way, the calibration standards run through the whole extraction procedure just like the real samples and analyte loss during extraction or liquid desorption is corrected for. Besides, there was no need for synthesizing the bis-oximes (which are not commercially available) as they were formed during the extraction/ derivatization process.

For each analyte, five calibration standards were prepared with spiked amounts of $\mathrm{G}$ (or MG) ranging from 1 to $60 \mathrm{ng}$. Blank measurements $(n=5)$ were conducted using $15 \mathrm{~mL}$ of ultrapure water to obtain values for the evaluation of the analytical limits and determine contamination levels of glassware, reagents, and stir bars.

\section{Sampling}

Snow samples were collected on a field next to the Meteorological Observatory at Hohenpeissenberg on Mount Hohenpeissenberg ( $977 \mathrm{~m}$ a.s.1., $47^{\circ} 48^{\prime} \mathrm{N}, 11^{\circ} 00^{\prime} \mathrm{E}$ ) in Bavaria, Germany. Preheated $500 \mathrm{~mL}$ glass sample flasks were filled with snow and sealed firmly using screw caps with PTFE-coated septa. Samples were collected in triplicate from the surface and from $30 \mathrm{~cm}$ depth.

Ice core samples were obtained from a $125 \mathrm{~m}$ long ice core from the upper Grenzgletscher, Monte Rosa massif (4,200 m a.s.l., $45^{\circ} 55^{\prime} \mathrm{N}, 7^{\circ} 52^{\prime} \mathrm{E}$ ) in the southern Swiss Alps. Drilling and storing conditions as well as glaciochemical dating of the ice core are described elsewhere (see [27, 28]). Samples from two different years (1959 and 1991) were analyzed as a proof of principle.

After collection, all samples were kept frozen and stored at $-20{ }^{\circ} \mathrm{C}$. On the day of analysis, the samples were thawed at room temperature and analyzed immediately to prevent any kind of decay.

\section{Results and discussion}

Method development

\section{Derivatization}

The SBSE efficiency of a particular analyte can be estimated using the octanol/water partitioning coefficient $\left(K_{O / W}\right)$ which is a reliable approximation for the partitioning coefficient between the PDMS phase and water $\left(K_{P D M S / W}\right)$. Together with the phase ratio ( $V_{\text {Water }} / V_{\text {PDMS }}$ ) the value of $\log K_{O / W}$ is used to determine the recovery of a certain analyte. Using commercially available stir bars with PDMS volume of $124 \mu \mathrm{L}$, only analytes with $\log K_{O / W}$ of $>3$ can be extracted with a recovery higher than $80 \%$ [26]. Because G and MG exhibit very low $\log K_{O / W}$ values $(-0.69$ and -0.58 for $\mathrm{G}$ and $\mathrm{MG}$, respectively), extraction of $G$ and MG with PDMS coating does not prove satisfactory. Therefore, derivatization was applied to convert the analytes into less polar bisoximes $\left(\log K_{O / W} 3.40\right.$ and 3.69 for G-bis-BHA and MG-bis-BHA, respectively). The $\log K_{O / W}$ values were calculated using ALOGPS $2.1[29,30]$.

Previous experiments also revealed that the analytes in their underivatized form showed poor chromatographic separation on a reversed phase HPLC column and low ionization efficiencies during ESI [23]. Conversion into larger and less polar compounds improved chromatographic properties and ionization efficiencies.

The most frequently used derivatization reagent for carbonyl compounds in combination with LC is DNPH. DNPH is soluble only in diluted strong mineral acids, but strong acidic conditions may damage the stir bar's PDMS coating. Hence, the water-soluble derivatization reagents PFBHA (O-(2,3,4,5,6-pentafluorobenzyl)hydroxylamine) and BHA (O-benzylhydroxylamine) were tested.

PFBHA is a common derivatization reagent for GC analyses and therefore available in high purity, which eliminates the need for purification. Nevertheless, both G and MG PFBHAderivatives showed strong variations in ionization efficiency with unacceptably high relative standard deviation (RSD) values up to $60 \%$ in triplicate measurements. Better results were obtained using BHA as derivatization reagent $(\mathrm{RSD}<$ $12 \%$ ), although purification is necessary because it is not commercially available in GC derivatization grade like PFBHA.

In the aqueous sample, the free form of $\mathrm{G}$ and $\mathrm{MG}$ is in equilibrium with hydrated monomeric and oligomeric forms. During derivatization, the derivatization reagent is used in excess (10,000 fold excess compared to a glyoxal concentration of $1 \mathrm{ng} / \mathrm{mL}$ ) and under acidic conditions ( $\mathrm{pH}$ 3.5) to ensure that the equilibrium is shifted towards the free monomeric form, which is accessible for the derivatization. That way, the measurements comprise the sum of free monomeric, hydrated, and reversibly formed higher oligomeric compounds [17, 31].

\section{Time course of extraction and liquid desorption}

Because SBSE is an equilibrium technique based on the partitioning of the analyte between the aqueous phase and the PDMS phase, the stirring times to reach equilibrium 
during extraction and liquid desorption were determined. Different extraction times $(1,2,5,10$, and $22 \mathrm{~h})$ were tested for both G- and MG-bis-oximes. A stirring speed of 1,000 rpm was used and liquid desorption parameters were set as follows: stirring time $3 \mathrm{~h}$, stripping solvent $1.5 \mathrm{~mL} \mathrm{ACN}$, stirring speed $500 \mathrm{rpm}$. As shown in Fig. 2a, in case of G, the equilibrium is reached after about $10 \mathrm{~h}$, whereas for $\mathrm{MG}$, a longer extraction time up to $22 \mathrm{~h}$ is needed. Therefore, an optimal extraction time of $20 \mathrm{~h}$ is used, which furthermore allows extraction overnight.

As the curve for the extraction of both analytes is similar, liquid desorption time was optimized using only MG. An extraction time of $20 \mathrm{~h}$, stripping solvent of $1.5 \mathrm{~mL} \mathrm{ACN}$ and stirring speed of $500 \mathrm{rpm}$ was applied. Liquid desorption times of $0.5,1,2,3,4,6,8$, and $20 \mathrm{~h}$ were tested. The equilibrium is reached after about $3 \mathrm{~h}$ with no significant change at longer stirring times, so $3 \mathrm{~h}$ is determined as optimal liquid desorption time (Fig. 2b).
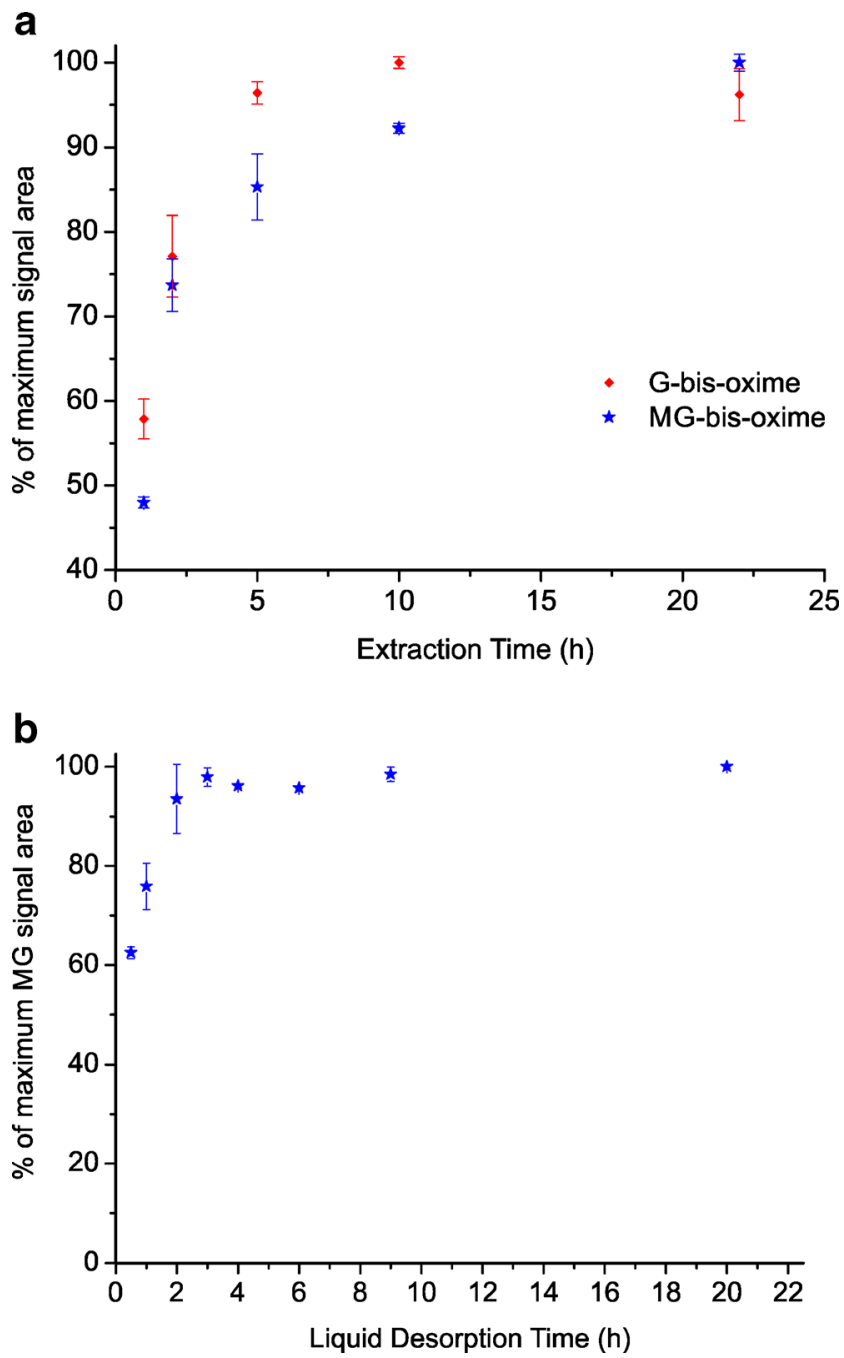

Fig. 2 Optimization of extraction time (a) and liquid desorption time (b)

\section{Liquid desorption solvent}

$\mathrm{MeOH}$ and $\mathrm{ACN}$ were tested as stripping solvents for liquid desorption. Using $\mathrm{ACN}$, higher signal areas of the bis-oximes were obtained, therefore ACN was chosen as the stripping solvent in further optimization steps. A volume of $1.5 \mathrm{~mL}$ is needed to completely immerse the stir bar in the stripping solvent and to ensure that it is covered while stirring. For the same reason, the stirring speed was adjusted to $500 \mathrm{rpm}$ during liquid desorption (instead of 1,000 rpm during extraction).

\section{Additional optimization}

Ionic strength and $\mathrm{pH}$ of the sample solution play an important role in the efficiency of the SBSE process. Derivatization is also influenced by $\mathrm{pH}$ which makes the process more complex. To ensure equal conditions for all samples and standards, they were acidified to a $\mathrm{pH}$ of about 3.5 with sulfuric acid prior to SBSE.

The influence of ionic strength on the extraction process was evaluated by adding $5-30 \mathrm{wt} \% \mathrm{NaCl}$ to the aqueous sample solutions. No significant change in SBSE efficiency was observed. Therefore, no $\mathrm{NaCl}$ addition was applied in the method.

\section{Sample volume}

The effect of different sample volumes on extraction efficiency was determined by spiking a distinct volume of a standard solution containing $\mathrm{G}$ and $\mathrm{MG}$ to different volumes (5$70 \mathrm{~mL}$ ) of ultrapure water. No significant differences in signal area were observed; hence, in the chosen volume range, the efficiency of the SBSE-LD process is independent of the sample volume, which can be adjusted depending on the expected concentration of the environmental sample.

\section{Chromatographic separation and $M S$}

The HPLC parameters were optimized for baseline separation and short retention times of G- and MG-bis-oximes. Separation was tested using $\mathrm{C} 18, \mathrm{C} 8$, and phenylhexyl columns. The best results based on peak shape and short retention time were obtained using a C8 column with gradient elution, heated to $30{ }^{\circ} \mathrm{C}$.

Electrospray and MS parameters were optimized for maximum signal intensity in the positive mode. Selectivity of the detection was enhanced using MRM. During an MRM cycle, a targeted parent ion is isolated in the ion trap mass analyzer, followed by CID (helium as collision gas) and isolation of a particular daughter ion from the resulting fragmentation spectrum. This daughter ion is used for quantification. The same cycle is successively repeated for the three parent ions $\mathrm{m} / \mathrm{z} 269$ 


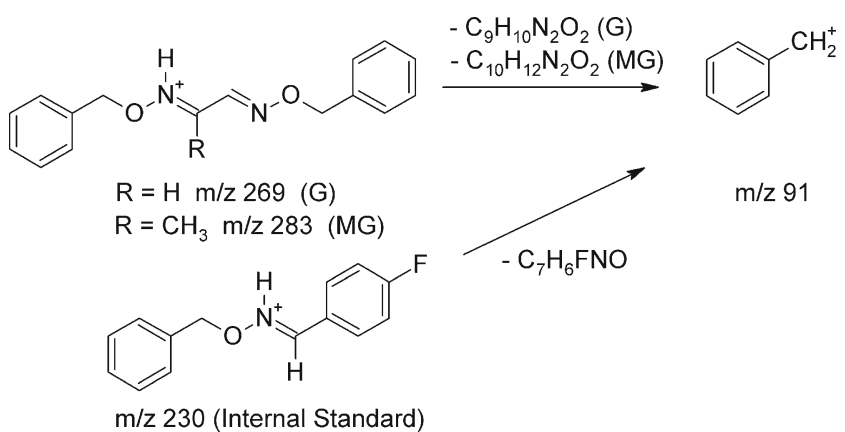

Fig. 3 Fragmentation of the precursor ions $[\mathrm{M}+\mathrm{H}]^{+}$of the analytes Gbis-oxime and MG-bis-oxime and the internal standard oxime to yield the product ion used for quantification

(G), $m / z 283$ (MG), and $\mathrm{m} / z 230$ (internal standard) multiple times during the chromatographic run. In initial MS/MS experiments, the fragment $\mathrm{m} / \mathrm{z} 91$ was identified as the most abundant product ion of both G- and MG-bis-oxime. Therefore $m / z 269 \rightarrow m / z$ 91, $m / z 283 \rightarrow m / z$ 91, and $m / z 230 \rightarrow m / z$ 91 (for $\mathrm{G}, \mathrm{MG}$, and internal standard) were used as product ion transitions in MRM mode (see Fig. 3).

\section{Validation}

Calibration functions for $\mathrm{G}$ and $\mathrm{MG}$ were obtained by linear regression analysis. Calibration parameters are summarized in Table 1. To determine the instrumental limits of detection and quantification, the standard deviation of the blank samples $(n=5)$ was multiplied by 3 and 10, respectively, and divided by the slope of the linear regression function. Instrumental limits of detection (ILOD) based on the amount of analyte in the liquid desorption extract were 242 and $213 \mathrm{pg} / \mathrm{mL}$ for $\mathrm{G}$ and MG. Method limits of detection and quantification based on a sample volume of $15 \mathrm{~mL}$ were 16 and $54 \mathrm{pg} / \mathrm{mL}$ for $\mathrm{G}$ and 14 and $47 \mathrm{pg} / \mathrm{mL}$ for $\mathrm{MG}$, respectively.

To determine the recovery of the analytes, snow samples were spiked with $\mathrm{G}$ and $\mathrm{MG}$ at three different concentration levels $(5-15 \mathrm{ng} / \mathrm{mL})$ and analyzed in triplicate. The masses of the spiked samples were corrected by subtracting the masses of the unspiked snow sample and then compared to the nominal spiked masses. The results of the different spike levels were averaged to determine the recovery of the respective target compound. Recoveries were $78.9 \pm 5.6 \%$ for $\mathrm{G}$ and $82.7 \pm 7.5 \%$ for MG, respectively. Method inter-batch precision was evaluated by calculation the RSD in percent from different triplicate analyses of snow and ice samples measured on different days. A precision of $7.2 \pm 4.9 \%$ for $\mathrm{G}$ and $11.4 \pm$ $5.9 \%$ for $\mathrm{MG}$ was determined.

Application

The performance of the described method was tested by analyzing fresh snow samples from Hohenpeissenberg, southern Germany, and ice core samples from Upper Grenzgletscher in the southern Alps, Switzerland. G was detected in both snow samples and ice core samples and MG was detected in the snow samples and one of the ice core samples. Snow samples were collected from the surface and from a layer in $30 \mathrm{~cm}$ depth in triplicate with a meltwater sample volume of $5 \mathrm{~mL}$ used for analysis. Concentrations (corrected for recovery) were $16.3 \pm 1.2 \mathrm{ng} / \mathrm{g}(280.8 \pm$ $20.7 \mathrm{nM})$ for $\mathrm{G}$ and $3.5 \pm 0.4 \mathrm{ng} / \mathrm{g}(48.6 \pm 5.6 \mathrm{nM})$ for $\mathrm{MG}$ in the surface snow samples. Similar concentrations were found in the layer $30 \mathrm{~cm}$ below the surface $(15.4 \pm 1.4 \mathrm{ng} / \mathrm{g}$ for $\mathrm{G}$ and $3.6 \pm 0.3 \mathrm{ng} / \mathrm{g}$ for $\mathrm{MG}$, respectively), indicating no age difference between the two layers. The obtained concentrations are in the same order of magnitude as concentrations reported in other field studies from urban and rural areas: 200-7100 nM for $\mathrm{G}$ and 30-60 $\mathrm{nM}$ for $\mathrm{MG}$ ([32], sampled in southern California), 44.8 and $25.0 \mathrm{nM}$ for $\mathrm{G}$ and MG in Tokyo snow ([21], converted from micrograms per liter values). Concentrations reported in this study are one order of magnitude higher than those found in remote, polar areas, i.e., 13.9 and $6.9 \mathrm{nM}$ for G and MG in Greenland Snow ([21]) and 13.937.1 and 1.9-6.5 $\mathrm{nM}$ for $\mathrm{G}$ and $\mathrm{MG}$ in surface snow from Barrow, Alaska ([17]). This can be explained by the much longer distance between polar regions and possible emission regions of $\mathrm{G}$ and $\mathrm{MG}$ or their precursors.

Ice core samples from two different years (sample 1: 1959, sample 2: 1991) were analyzed. Resulting concentrations were $0.178 \mathrm{ng} / \mathrm{g}(3.06 \mathrm{nM})$ for $\mathrm{G}$ and $0.126 \mathrm{ng} / \mathrm{g}(1.75 \mathrm{nM})$ for $\mathrm{MG}$ in sample 1 and $0.085 \mathrm{ng} / \mathrm{g}(1.46 \mathrm{nM})$ for $\mathrm{G}$ in sample 2 with MG below detection limit. The meltwater sample volume used for analysis was $15 \mathrm{~mL}$. The concentration values are in the same order of magnitude as reported in a field study focusing on the chemical composition of a Greenland ice core $(0.023-1.14 \mathrm{ng} / \mathrm{g}$ for $\mathrm{G}$ and $0.031-1.36 \mathrm{ng} / \mathrm{g}$ for MG, [19]).

The concentrations measured in the ice core are much lower (almost two orders of magnitude for $G$ ) than the

Table 1 Calibration data

\begin{tabular}{|c|c|c|c|c|c|c|}
\hline & Slope $^{\mathrm{a}}$ & Intercept $\mathrm{t}^{\mathrm{a}}$ & $R^{2}$ & Recovery (\%) & ILOD (ng/mL) & ILOQ (ng/mL) \\
\hline G & 0.037 & -0.018 & 0.9988 & $78.9 \pm 5.6$ & 0.242 & 0.807 \\
\hline $\mathrm{MG}$ & 0.025 & -0.034 & 0.9941 & $82.7 \pm 7.5$ & 0.213 & 0.711 \\
\hline
\end{tabular}

${ }^{a}$ Determined using the measured signal area quotient (analyte/internal standard) 
concentrations in the fresh snow samples. Obviously, as this is only a limited dataset, it is not possible to draw reliable conclusions regarding the fate of $\mathrm{G}$ and $\mathrm{MG}$ in the snow or ice samples; however, the described analyses show the good applicability of the method to the given issue of detecting small and even trace amounts of G and MG in snow and ice core samples.

\section{Conclusions}

We have described the development and optimization of a new, sensitive, and selective method using stir bar sorptive extraction with liquid desorption and HPLCMS/MS to quantify the alpha-dicarbonyls glyoxal and methylglyoxal in molten ice and snow. Key SBSE parameters such as extraction and liquid desorption time, derivatization, salt addition, and desorption solvent were optimized. Method detection limits were in the low picogram per milliliter range. The method was successfully applied to snow and ice core samples from the Alpine region. For snow samples, a sample volume of only $5 \mathrm{~mL}$ is sufficient; for less concentrated samples like ice cores, the sample volume can be adjusted to larger volumes (i.e., $15 \mathrm{~mL}$ ) without loss of extraction efficiency.

Analyses of snow samples from different depth and location may give hints for the interaction of $G$ and $M G$ between snow and atmosphere. A larger dataset is needed improve the understanding of the complex processes occurring in the snowpack. Historic records of G (and MG) in ice cores can give hints concerning the oxidative capacity of the atmosphere, as the analytes are tracers of VOC oxidation chemistry and SOA. Besides, ice core data may be of great benefit especially in connection with other marker compounds of biogenic and anthropogenic origin to get information on qualitative and quantitative changes of emission sources over time.

\section{References}

1. Fu T, Jacob D, Wittrock F, Burrows J, Vrekoussis M, Henze D (2008) Global budgets of atmospheric glyoxal and methylglyoxal, and implications for formation of secondary organic aerosols. J Geophys Res 113(D15):D15303

2. Mahowald N, Ward DS, Kloster S, Flanner MG, Heald CL, Heavens NG, Hess PG, Lamarque J, Chuang PY (2011) Aerosol impacts on climate and biogeochemistry. In: Gadgil A, Liverman DM (eds) Annu Rev Environ Resour 36:45-74

3. Kawamura K, Steinberg S, Kaplan IR (1996) Concentrations of monocarboxylic and dicarboxylic acids and aldehydes in southern California wet precipitations: Comparison of urban and nonurban samples and compositional changes during scavenging. Atmos Environ 30(7):1035-1052
4. Carlton A, Wiedinmyer C, Kroll JH (2009) A review of secondary organic aerosol (SOA) formation from isoprene. Atmos Chem Phys 9:4987-5005

5. van Pinxteren M, Herrmann H (2013) Glyoxal and methylglyoxal in Atlantic seawater and marine aerosol particles: method development and first application during the Polarstern cruise ANT XXVII/4. Atmos Chem Phys 13(23):11791-11802

6. Carlton AG, Turpin BJ, Altieri KE, Seitzinger S, Reff A, Lim H, Ervens B (2007) Atmospheric oxalic acid and SOA production from glyoxal: results of aqueous photooxidation experiments. Atmos Environ 41(35):7588-7602

7. Matsunaga SN, Guenther AB, Izawa Y, Wiedinmyer C, Greenberg JP, Kawamura K (2007) Importance of wet precipitation as a removal and transport process for atmospheric water soluble carbonyls. Atmos Environ 41(4):790-796

8. Kawamura K, Kasukabe H, Barrie L (1996) Source and reaction pathways of dicarboxylic acids, ketoacids and dicarbonyls in arctic aerosols: one year of observations. Atmos Environ 30(10):17091722

9. Ervens B, Volkamer R (2010) Glyoxal processing by aerosol multiphase chemistry: towards a kinetic modeling framework of secondary organic aerosol formation in aqueous particles. Atmos Chem Phys 10(17):8219-8244

10. Altieri K, Seitzinger S, Carlton A, Turpin B, Klein G, Marshall A (2008) Oligomers formed through in-cloud methylglyoxal reactions: chemical composition, properties, and mechanisms investigated by ultra-high resolution FT-ICR mass spectrometry. Atmos Environ 42(7):1476-1490

11. Dominé F, Shepson PB (2002) Air-snow interactions and atmospheric chemistry. Science 297:1506-1510

12. Ariya PA, Domine F, Kos G, Amyot M, Côté V, Vali H, Lauzier T, Kuhs WF, Techmer K, Heinrichs T, Mortazavi R (2011) Snow - a photobiochemical exchange platform for volatile and semi-volatile organic compounds with the atmosphere. Environ Chem 8(1):62-73

13. Sieg K, Fries E, Püttmann W (2008) Analysis of benzene, toluene, ethylbenzene, xylenes and n-aldehydes in melted snow water via solid-phase dynamic extraction combined with gas chromatography/mass spectrometry. J Chromatogr A 1178(1-2):178-186

14. Lacorte S, Quintana J, Tauler R, Ventura F, Tovar-Sánchez A, Duarte C (2009) Ultra-trace determination of persistent organic pollutants in Arctic ice using stir bar sorptive extraction and gas chromatography coupled to mass spectrometry. J Chromatogr A 1216(49):8581-8589

15. Winterhalter R, Kippenberger M, Williams J, Fries E, Sieg K, Moortgat GK (2009) Concentrations of higher dicarboxylic acids C-5-C-13 in fresh snow samples collected at the high alpine research station Jungfraujoch during CLACE 5 and 6. Atmos Chem Phys 9(6):2097-2112

16. Matsunaga S, Kawamura K (2000) Determination of $\alpha$ - and $\beta$ hydroxycarbonyls and dicarbonyls in snow and rain samples by GC/FID and GC/MS employing benzyl hydroxyl oxime derivatization. Anal Chem 72(19):4742-4746

17. Houdier S, Barret M, Dominé F, Charbouillot T, Deguillaume L, Voisin D (2011) Sensitive determination of glyoxal, methylglyoxal and hydroxyacetaldehyde in environmental water samples by using dansylacetamidooxyamine derivatization and liquid chromatography/ fluorescence. Anal Chim Acta 704(1-2):162-173

18. Douglas TA, Domine F, Barret M, Anastasio C, Beine HJ, Bottenheim J, Grannas A, Houdier S, Netcheva S, Rowland G, Staebler R, Steffen A (2012) Frost flowers growing in the Arctic ocean-atmosphere-sea ice-snow interface: 1 . Chemical composition. J Geophys Res 117:D00R09

19. Kawamura K, Yokoyama K, Fujii O, Watanabe O (2001) A Greenland ice core record of low molecular weight dicarboxylic acids, ketocarboxylic acids, and alpha-dicarbonyls: a trend from little 
ice age to the present (1540 to 1989 A.D.). J Geophys Res 106(D1): $1331-1345$

20. Bao M, Pantani F, Griffini O, Burrini D, Santianni D, Barbieri K (1998) Determination of carbonyl compounds in water by derivatization-solid-phase microextraction and gas chromatographic analysis. J Chromatogr A 809(1-2):75-87

21. Kawamura K (1993) Identification of C2-C10 w-oxocarboxylic acids, pyruvic acid, and alpha-dicarbonyls in wet precipitation and aerosol samples by capillary GC and GC/MS. Anal Chem 65:3505-3511

22. Xu F, Zou L, Liu Y, Zhang Z, Ong CN (2011) Enhancement of the capabilities of liquid chromatography-mass spectrometry with derivatization: general principles and applications. Mass Spectrom Rev 30(6):1143-1172

23. Kampf CJ, Bonn B, Hoffmann T (2011) Development and validation of a selective HPLC-ESI-MS/MS method for the quantification of glyoxal and methylglyoxal in atmospheric aerosols (PM2.5). Anal Bioanal Chem 401(10):3115-3124

24. Wang H, Zhang X, Chen Z (2009) Development of DNPH/HPLC method for the measurement of carbonyl compounds in the aqueous phase: applications to laboratory simulation and field measurement. Environ Chem 6:389-397

25. Neng N, Cordeiro C, Freire A, Nogueira J (2007) Determination of glyoxal and methylglyoxal in environmental and biological matrices by stir bar sorptive extraction with in-situ derivatization. J Chromatogr A 1169(1-2):47-52
26. David F, Sandra P (2007) Stir bar sorptive extraction for trace analysis. J Chromatogr A 1152(1-2):54-69

27. Eichler A, Schwikowski M, Gäggeler HW, Furrer V, Synal H, Beer J, Saurer M, Funk M (2000) Glaciochemical dating of an ice core from upper Grenzgletscher (4200 m a.s.1.). J Glaciol 46(154):507-515

28. Eichler A, Schwikowski M, Gäggeler HW (2000) An Alpine ice-core record of anthropogenic $\mathrm{HF}$ and $\mathrm{HCl}$ emissions. Geophys Res Lett 27(19):3225-3228

29. Tetko IV, Gasteiger J, Todeschini R, Mauri A, Livingstone D, Ertl P, Palyulin VA, Radchenko EV, Zefirov NS, Makarenko AS, Tanchuk VY, Prokopenko VV (2005) Virtual computational chemistry laboratory_design and description. J Comput Aided Mol Des 19(6): 453-463

30. Virtual Computational Chemistry Laboratory VCCLAB (2005) http://www.vcclab.org. Accessed 01.08.2013

31. Kampf CJ, Corrigan AL, Johnson AM, Song W, Keronen P, Königstedt R, Williams J, Russell LM, Petäjä T, Fischer H, Hoffmann T (2012) First measurements of reactive $\alpha$-dicarbonyl concentrations on $\mathrm{PM}_{2.5}$ aerosol over the Boreal forest in Finland during HUMPPA-COPEC 2010 - source apportionment and links to aerosol aging. Atmos Chem Phys 12(14):6145-6155

32. Gunz DW, Hoffmann MR (1990) Field investigations on the snow chemistry in central and southern California - II. Carbonyls and carboxylic acids. Atmos Environ 24A(7):1673-1684 